\title{
3D Full-Wave modelling and mode conversion in realistic $\mathrm{W} 7-\mathrm{X}$ plasmas
}

\author{
Pavel Aleynikov, Nikolai B. Marushchenko
}

Max-Planck-Institut für Plasmaphysik, Greifswald, Germany, pavel.aleynikov@ipp.mpg.de

Electron Cyclotron Resonance Heating (ECRH) is the main plasma heating mechanism in Wendelstein 7-X (W7-X) Stellarator. It is provided by 10 gyrotrons at $140 \mathrm{GHz}$ (corresponding to second-harmonic resonance at $2.5 \mathrm{~T}$ ) with the power of $1 \mathrm{MW}$ each.

Microwaves, launched from a vacuum, are reflected from their corresponding cut-off layers. In W7-X, the X2 and $\mathrm{O} 2$-modes are used in a wide range of operation scenarios. Whereas $\mathrm{X} 2$ has a cut-off at $1.2 \times 10^{20} \mathrm{~m}^{-3}$, the O2-mode can be used in plasmas with densities up to $2 \times 10^{20} \mathrm{~m}^{-3}$. The propagation of these modes is well tested experimentally and studied theoretically within geometrical optics (WKB).

Possible operation at higher densities would involve mode-conversion (from $\mathrm{O}$ - to $\mathrm{X}$ - to Bernstein-mode, i.e. "OXB"). The conversion of modes is outside of the applicability of WKB-theory and usually considered with the "full-wave" approach. Full-wave models are also used to study processes which involve scales comparable to the wavelength $(\sim 1 \mathrm{~mm})$, for example, effects of the density fluctuations.

This work reports on the development of a new 3D cold plasma full-wave code. The code utilizes the Finite Difference Time Domain (FDTD) technique [1] and has an interface with the ray-tracing code TRAVIS [2]. The computation domain is "minimized" around the WKBtrajectory obtained from the ray-tracing code; the background magnetic field is recovered from the precomputed 3D equilibrium data. The code takes advantage of massive parallel computations with Graphics Processing Units (GPUs), which allows for up to $\times 100$ faster calculations than on a single-CPU.

The minimization of the computation domain plays a critical role in efficient 3D computations. A so-called CPML boundary layer [3] is implemented in our fullwave code to truncate the computation domain. Note that is it common to discretize the magnetized plasma response current field $(\mathbf{J})$ in such a way that its $J_{x}, J_{y}$ and $J_{z}$ components are co-located in space $[1,4,5]$. This facilitates the current update calculation. However, we find the stability of such a scheme to be unsatisfactory, in particular when applied to the CMPL region. Therefore we have implemented a slightly more computationally demanding but more stable scheme where $\mathbf{J}$ is discretized in the same way as the wave electric field $(\mathbf{E})$. With this scheme, the performance of the CPML layer is found to be satisfactory.

One of the applications of our code is the calculation of the $\mathrm{O}$ - to-X-mode conversion efficiency in 3D plasmas.

Figure 1 shows an example of a full-wave 2D calculation of the $\mathrm{O}-\mathrm{X}$ conversion process in a realistic $\mathrm{W} 7-\mathrm{X}$ plasma. The O-mode, launched from vacuum (bottom right corner of the left plot), propagates into the plasma and partly reflects off the corresponding cut-off layer, where a part of the O-mode is converted into a slow Xmode, which propagates toward the upper-hybrid resonance. Eventually, this $\mathrm{X}$-mode will be converted to
Bernstein wave. The reflected part of the O-mode has a "hole" (due to the non-uniform $\mathrm{O}-\mathrm{X}$ conversion). The perpendicular component of the refractive index is computed with the use of a windowed Fourier transform and shown in Fig. 1 (right). The incoming O-mode, the reflected O-mode, and the slow-X mode (which settles near the Upper Hybrid resonance layer) can be clearly distinguished in this diagram, which appears to be in an agreement with expectations from WKB theory (i.e. dispersion curves). Note that the slow-X mode is eventually dissipated numerically without leaving the computation domain. This dissipation is of purely numerical nature. It happens when the wavelength of the mode becomes shorter than $\sim 10$ computational cells.

The W7-X plasma is strongly shaped and requires a $3 \mathrm{D}$ evaluation of the conversion process. In Fig. 2 an example of such a $3 \mathrm{D}$ calculation of the above scenario is shown (the wave is launched from the bottom of the domain). The decimal logarithm of the time-averaged $\mathrm{E}^{2}$ is color-coded. The sequence of dark-colored points represents the corresponding WKB trajectory. It is evident from the cross-section of the reflected wave that the $3 \mathrm{D}$ geometry needs to be appropriately accounted for.

Thanks to its speed, the GPU-capable 3D FDTD code allows for efficient large parametric scans over a broad range of parameters.

To estimate the power conversion efficiency, we compute surface integrals of the time-averaged Poynting vector (i.e. the intensity) over every boundary of the computation domain:

$$
S_{\mathrm{i}}=\left(1 / \mu_{0}\right) \iint(<[\mathbf{E} \times \mathbf{B}]>\bullet \mathbf{n}) d s,
$$

where the index "i" denotes a particular boundary, n represents its normal vector and $\langle\ldots\rangle$ denotes time averaging. In the above case, the OX conversion efficiency can be calculated as the difference between the intensities of the input $\left(S_{i n}\right)$ and reflected $\left(S_{r e f}\right)$ waves.

An estimate of the OX conversion efficiency is made as follows for W7-X-relevant ECRH beam parameters and plasma geometry. A Gaussian beam approximation is used as an initial condition. A beam of $1.5 \mathrm{~cm}$ in radius and a phase front curvature of $1 \mathrm{~m}$ (i.e. beam waist of radius $1.4 \mathrm{~cm}$ located near the $\mathrm{O}$-mode cut-off) approximates the ECRH beam at the plasma entrance. The density profile is chosen arbitrarily (with normalized gradient of $\sim 20 \mathrm{~m}^{-1}$ near the cutoff). The antenna launcher location was fixed, corresponding to the upper port "A, C1" in W7-X nomenclature. Then, the launcher azimuth and altitude angles were varied to find the highest OX conversion conditions. Contours of the calculated OX conversion fraction $\left(S_{i n}+S_{\text {ref }}\right) / S_{\text {in }}$ are shown in Fig. 3.We find that for the given conditions (equilibrium, density profile, launcher position and beam focusing) the maximum conversion reaches $48 \%$.

The analogous 2D calculations result in different contours of the conversion fraction (see Fig. 4). This is due to the fact that the truncation from $3 \mathrm{D}$ to $2 \mathrm{D}$ is 


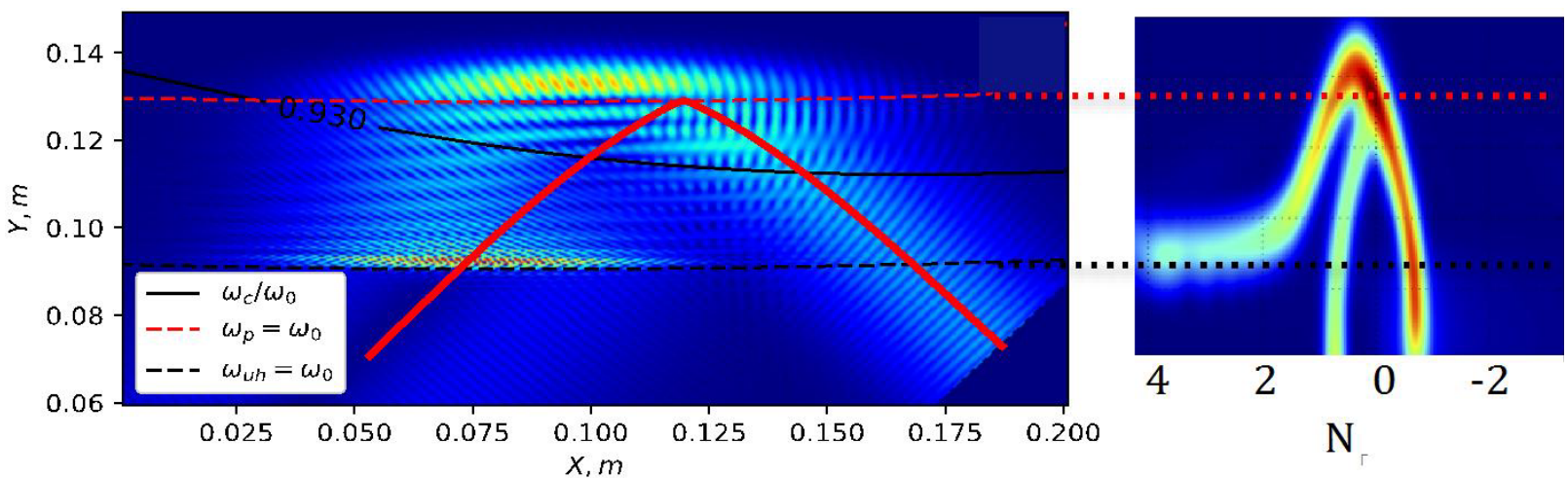

Fig. 1. An example of the calculation of the O-X mode conversion in realistic W7-X plasma. Left plot: the snapshot of the electric field amplitude (color-coded), ray tracing (solid red), antenna is in the right bottom corner. O-mode cutoff and Upper Hybrid resonance layer are plotted with the red and black dashed curves, respectively. Right plot: windowed Fourier transform of the wave field (wave refractive index), window is moved along the $\mathrm{Y}$ axis (the plots have common $\mathrm{Y}$ axis)

somewhat arbitrary in the general case: the computational domain is constructed in such a way that the distance from the ray-tracing trajectory to the computation plane is minimized. While this approximation still allows for good agreement between the ray-tracing trajectory and the full-wave solution for the reflected O-mode, the conversion fraction changes significantly (the maximum reaches $60 \%$ ).

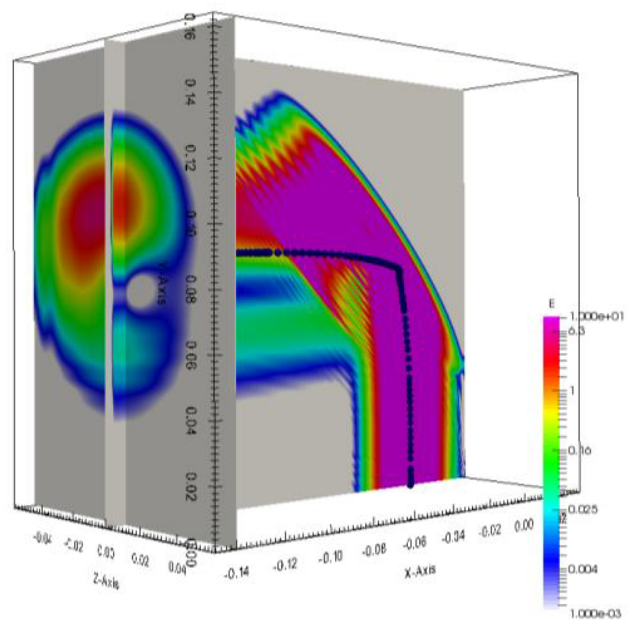

Fig. 2. 3D calculation of the scenario described in Fig. 1

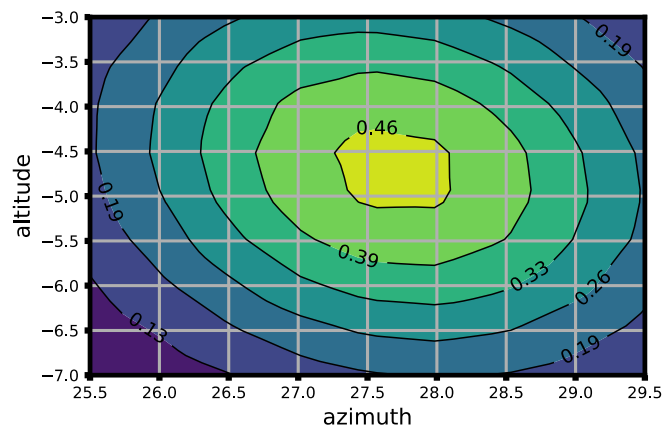

Fig. 3. Contours of the OX conversion efficiency for a given plasma parameters over varying beam aiming angles (for a fixed location of the launcher)

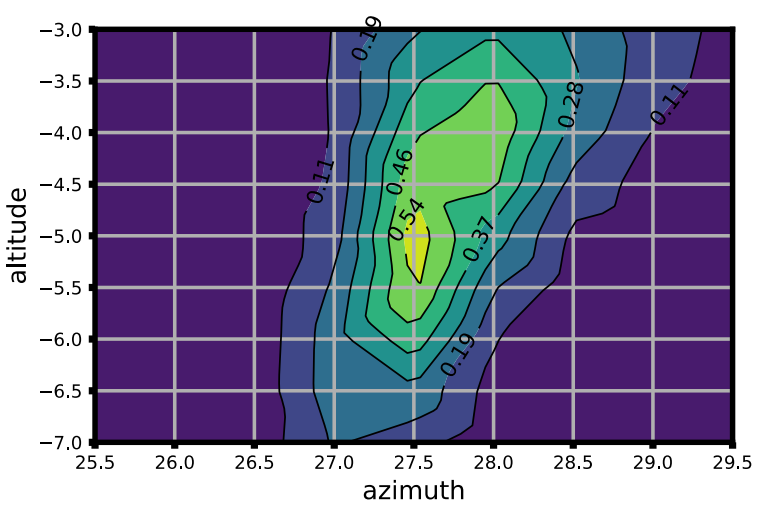

Fig. 4. Same as Fig. 3 for $2 \mathrm{D}$ calculations

These results do not yet form a comprehensive picture of the optimal conditions for OXB heating in W7-X, but such a study is planned in future. Other future work includes: comparison of the results with analytical 3D OX conversion models [6, 7]; study of the X-O conversion for the needs of EBE diagnostics; and a study of the effects of plasma fluctuations on beam propagation.

In summary we conclude that the newly developed GPU-capable 3D FDTD code allows for a very efficient assessment of wave physics in realistic 3D plasmas.

\section{References}

1. Inan, U., Marshall, R. // Numerical electromagnetics: the FDTD method, Cambridge, 2011.

2. Marushchenko N., Turkin, Y., Maassberg, H. // Computer Physics Communications. 2014. V. 185. P. 165.

3. Roden J, Gedney S.D. // MICROWAVE AND OPTICAL TECHNOLOGY LETTERS. 2000. V. 27. P. 5.

4. Kohn, A., et al. Plasma Phys. Control. Fusion. 2008. V. 50. P. 085018.

5. Williams, T., Kohn, A., O’Brien M., Vann R. // Plasma Phys. Control. Fusion, 2014, V. 56 P. 075010.

6. Gospodchikov E., Khusainov T., Shalashov A. // Plasma Phys. Control. Fusion, 2012, V. 54 P. 045009.

7. Popov A. // Plasma Phys. Control. Fusion, 2011, V. 53 P. 065016. 